\title{
Philosophical Reflections of a Starch Chemist
}

\author{
By Thomas J. Schoch \\ College of Home Economics, Cornell University, Ithaca, New York, U.S.A.
}

In one way or another, I have been deeply involved in starch chemistry for the past 39 years. Most of this work has been interesting, and some of it even exciting. But perhaps as a psychological rejection of this narrow specialization, I find myself confronted with certain questions of a very general nature:

What is the real purpose of scientific research?

What is the best criterion of accomplishment in research?

How can facts be differentiated from fancies?

Are any of our scientific concepts permanently valid?

I would like to offer a somewhat skeptical and irreverent viewpoint of what science is and how it got to be that way. I ask your indulgence to wander off occasionally into the misty fog of philosophical and historical speculation, with the promise that I will try to land up with both feet on solid ground. Much of my illustrative material will be drawn from the field of starch chemistry, with a few assisting observations from another favorite of mine, science fiction.

Living organisms contain three principal types of polysaccharides, derived in large part from the sugar glucose:

Glucose itself is the instant energy fuel in the bloodstream of animals. It is also the principal intermediate for growth in the plant, photosynthesized in green-leaf tissue, and then conveyed via the sap to the regions of active growth.

Cellulose is the structural building material of plants, consisting of a waterinsoluble linear-chain polymer of glucose. The linkages between the individual glucose units are such that the cellulose molecule is extended and rigid, in order to provide structurál strength.

Starch is likewise a glucose polymer, usually stored as microscopically-small granules in the seeds and roots of plants. It is more soluble than cellulose, and serves as a slowly available food supply for the plant during periods of dormancy or germination, when there are no green leaves or no sunlight to synthesize glucose. Most starches contain two types of glucose polymers: (1) a linear-chain molecule with flexible linkages between the glucose units, so that the chain can twist and coil like cooked spaghetti, and (2) a loosely branched or tree-like polymer of glucose.

Glycogen is a water-soluble polymer of glucose with a bush-like structure, much more tightly branched than the branched starch fraction. It serves principally as a readily available form of reserve glucose in animals, being stored in the liver and also in the muscle tissue. When the animal expends energy or is subjected to strong fears which deplete blood sugar, this glycogen is rapidly enzyme-hydrolyzed to 
glucose. Thus a scared animal can use up a substantial portion of its stored glycogen within a minute.

So far, everything is clear and simple and orderly. But immediately a host of unanswerable questions and inexplicable complexities arise to muddy the water:

1. Each kind of starch has granules of characteristic size and shape, with very wide variations between species. Thus we have the tiny one-micron granules from Polynesian taro root as contrasted with the enormous granules of canna starch. Or the spherical granules of rye starch as compared with the peculiar composite granules from wrinkled-seeded peas. Is any useful purpose served by this extreme diversity in size and form of the granules? Or are these merely meaningless accidents which inexplicably became fixed in the genetic pattern of the plant?

2. Most starches (such as corn, wheat and rice) contain about $25 \%$ of linear glucose polymer and $75 \%$ of branched polymer. However, the starches from certain so-called waxy cereals contain only branched fraction, while the starches from highamylose corn and pea are predominantly linear polymer. Why these differences in polysaccharide structure, which seem quite unrelated to the function of starch as reserve carbohydrate supply?

3. While ordinary dent corn stores only starch in the kernel, sweet corn contains starch, glycogen and sugar. Why this mixture? And why does a slow-germinating seed need rapidly assimilable glycogen and sugar in its food storage depot?

4. Medical biochemists have recently identified a glycogen-storage disease in humans, whereby the glycogen becomes less highly branched and more like the branched fraction of starch. This can become dangerous because the body cannot quickly draw on such a reserve carbohydrate. Why this disorder in which the human system manufactures plant-like carbohydrates? Could this be a sort of biological "memory," by which the animal cell reverts back through countless millennia and thus behaves like its ancient ancestor, the plant cell?

Now it might be hoped that carbohydrate science would rationalize such natural phenomena, to reduce them to simple understandable cause-and-effect principles. After all, the word "science" (according to the dictionary) means the systematized knowledge of demonstrable truths and the formulation therefrom of general laws. Hence science should not be a wildly heterogeneous mass of unrelated and indigestible facts, but rather should lead to an orderly set of laws governing and relating these facts. This was perhaps true of science some 50 or 100 years ago; it no longer appears to be true today. As science has become infinitely more extensive and ramified, so the explanations offered by science have become increasingly clumsy and complicated and irrational, to the point where they frustrate rather than satisfy the human intelligence. Thus it seems today that science develops complexity and disorder far more rapidly than simplicity and order. To take an example from atomic physics, originally only two kinds of elemental particles were postulated: negatively-charged electrons and positively-charged nuclei. Now we have a whole slew of subatomic particles, some of them so off-beat that they had to be dreamed up by the science-fiction writers before the scientist could "discover" them. For 
instance, anti-matter, where the nucleus is negative and the electrons positive. Or recent reports that certain outer-space "bodies" may consist of compacted atomic nuclei, with a density corresponding to billions of tons per cubic inch. Or the concept that time may run backward in a contracting universe. What do we mean by "backward"? How can we conceive such a thing?

This phantasmagoria has something in common with the snakes and pink elephants of an alcoholic delirium. I get a strong feeling that the complexities of science are limited only by the human imagination, and that anything which can be clearly conceived by the mind eventually becomes possible in fact. This notion isn't original: indeed, it was expressed by Descartes in 1636. Too many ideas have progressed through the successive stages of impossible to improbable to conceivable to plausible to possible to factual. To cite an example of this progression of concepts, for some thousands of years it was considered impossible that man could fly through the air except by the aid of witchcraft or magic. Then came practical dreamers like Leonardo da Vinci, who studied the wing structure of birds and tried to understand the mechanics of flight. So today we fly, quickly and effortlessly. And perhaps within the next decade, for some foolish and ill-defined reason, man may actually venture on a round-trip voyage to the moon. To probe even deeper into outer space, we must somehow circumvent the axiom that material objects cannot approach or exceed the speed of light. Here the science-fiction writers have led the way in imaginative thinking, primarily because their space-rocketing heroes could not pack enough miles or light-years into one lifetime to go gallivanting around among the galaxies. So today there are presumably sane individuals who talk of "hyperspace" and even of supra-light speeds, who are neither burned at the stake nor incarcerated in the nearest insane asylum. Indeed, they may even be scientists employed at U.S. space research centers.

Starch chemistry offers numerous examples of this waxing and waning of concepts, for example, as related to the structure of the starch granule and the mechanism of swelling. The tiny particles or granules of starch are insoluble and unchanged in cold water. But when heated in water medium above a certain critical temperature, they undergo a progressive swelling until the swollen granules have absorbed all the free water in the system and hence begin to crowd one another. And so we get a thick-bodied paste chock-full of these swollen granules. This phenomenon (which we call "gelatinization") occurs in a loaf of bread during baking, or during the cooking of a starch-thickened sauce or cream soup. There is no doubt what happens, since we can watch this swelling under the microscope. The enigma lies in HOW and WHY it happens_-matters of mechanism and theory-, and this problem has interested starch chemists for well over a hundred years.

Some 70 years ago, an eminent German botanist, Arthur Meyer by name, noted that starch granules appeared to have a crystalline organization-that is, they seemed to be composed of structural elements in some sort of orderly arrangement. For one thing, the granule showed a strong interference cross when viewed under the microscope with polarized light, a phenomenon indicative of crystallinity. 
Also, when the granule was crushed by pressure on the microscope cover glass, it tended to fracture along radial cleavage lines. These observations suggested to the botanist two possible structures: (1) concentric growth lamellations, like an onion, (2) radiating trichites or needles, like a sycamore button ball or the seeds on a dandelion head. Arthur Meyer combined both ideas into one theory, postulating

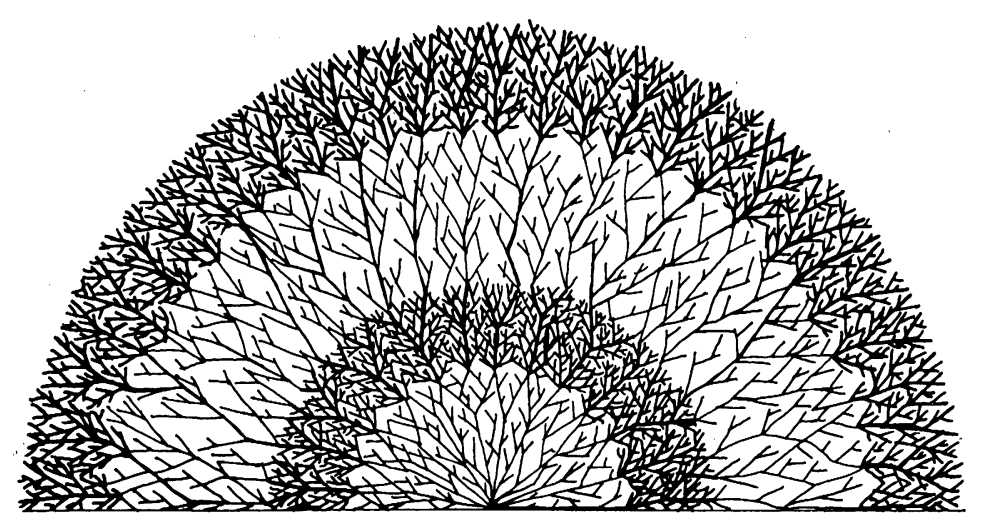

Fig. 1. The starch spherocrystal as originally conceived by the botanist Arthur Meyer in 1895. Branching trichitic crystals were pictured in radial orientation, and concentric lamellations in the granule were attributed to alternate shells of dense and loose packing.
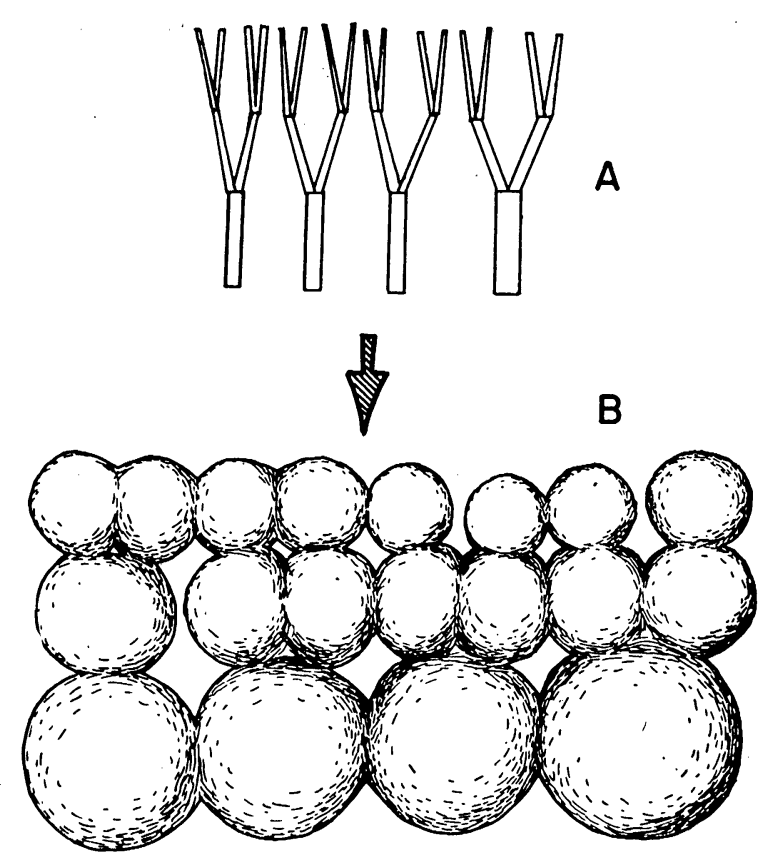

Fig. 2. Mechanism of swelling of the starch granule as portrayed by Arthur Meyer in 1895. Individual trichitic segments (A) imbibe water and swell laterally to give bulbous hydrated crystals (B). 
that the starch granule was made up of layers or growth-rings like a tree (Fig. 1). In each such layer were branching crystal needles of starch substance arranged in radial fashion. When the granule swelled in hot water (Fig. 2), this was merely the lateral lobe-like swelling of these crystal needles as they soaked up water. It was a nice theory, and had a certain appeal from a mechanical and botanical standpoint. But it never got outside the botanical laboratory, probably because the idea had no apparent practical or economic significance. I will expand somewhat on this point later.

Around 1910, the scientific world discovered colloid chemistry, and the concept of the semi-permeable membrane became the explanation for virtually every biochemical phenomenon. Such a membrane would allow water to pass quite freely, but would hold back such colloids as starch and gelatin. Thus the starch granule was conceived as an outer membrane or skin enclosing the interior water-soluble starch substance. Water progressively penetrated through this membrane and dissolved the interior starch, eventually giving a distended sac or envelope full of viscous starch solution. This swollen sac theory had little or nothing to recommend it, though the idea had persisted in the starch literature for 50 years and is still encountered in many high-school and college textbooks.

The early 1930's saw the birth of a high-polymer chemistry, which has yielded such products as nylon, polyethylene, and vinyl plastics. One of the earliest of the high-polymer chemists was Kurt Meyer* (not related to the earlier Arthur), who had made a considerable study of cellulose and then carried his ideas over to starch. Perhaps the most important of his concepts concerned the attractive forces between long linear-chain molecules which are adjacent and parallel in space. If these linear molecules contain hydroxyl groups, the intermolecular bonding becomes very strong. Now cellulose is a rigid linear molecule composed of glucose units, each of which has three hydroxyl groups. Consequently, many cellulose chains are associated together into bundles, which in turn make up the fibrils of wood structure or cotton filaments. While the linear starch molecule is much more flexible than cellulose, it likewise is prone to associate laterally with other chains. Hence Kurt Meyer pictured the starch granule as composed of lamellar layers of starch substance. In each such layer, the intermingled and intertangled linear and branched molecules are presumably laid down in radial fashion by growth processes (Fig. 3). Wherever linear chains or linear segments of branched molecules parallel one another, they associate together into crystalline bundles similar to those in cellulose. Between these areas, the molecules are arranged more haphazardly, with little or no association between them. When the starch granule is heated in water, these nonassociated regions hydrate and try to dissolve, with consequent expansion of the granule. But the total structure is still held together and dissolution prevented by the persistence of the associated regions. So eventually, the granule swells to an

* On one of his two visits to the United States, Kurt Meyer told me that, as a small boy, he frequently visited the courtyard of Arthur Meyer to watch the goldfish in an outdoor pool. $\mathrm{He}$ commented on the kindliness of his older namesake. 


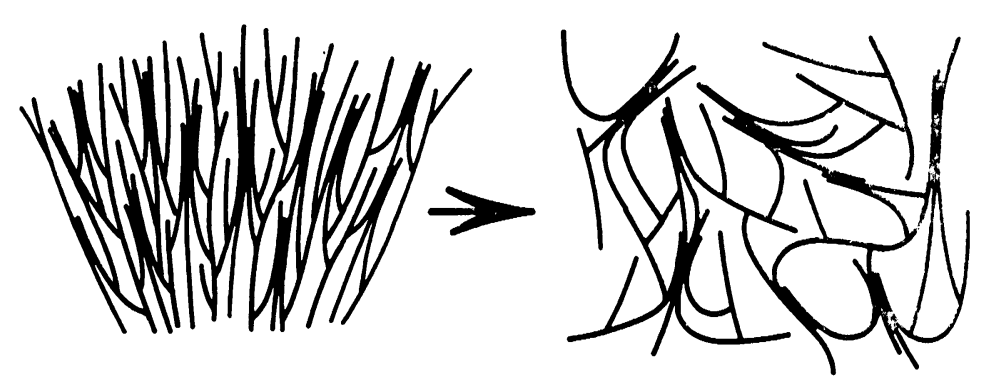

\section{UNSWOLLEN SEGMENT}

\section{SWOLLEN SEGMENT}

Fig. 3. Mechanism of swelling of granular starch. Regions of association shown as thickened sections.

expanded network of interlacing molecules, spot-welded together here and there by these persistent regions of crystallinity.

This concept has provided a very satisfactory working hypothesis for the past 25 years, and has greatly stimulated both fundamental and practical starch research. But we are now beginning to realize that the theory has flaws and discrepancies, and that it can no longer answer our questions about starch behavior. These inadequacies in the Kurt Meyer model will continue to multiply, until some new and totally different scheme is concocted to explain the starch granule and its mechanism of swelling. Micellar bundles are demonstrably valid for cellulose, but not for starch. I myself would prefer the simpler concept that associative bonding within the granule is exerted between pairs of linear chains, either sections of the linear fraction or outer branches of the branched fraction. Since the strength of this associative bonding is directly related to the length of the paired section, the first stages of gelatinization would represent dissociation of the shorter pairs, and longer pairs would persist to a higher temperature.

Since this talk is intended to be more speculative than scientific, permit me to digress again with some philosophical comments. We have been led to believe that science is a seeker after truth, and that somewhere there must be a perfect and permanently satisfactory concept of the starch granule. As ever-hopeful experimenters, perhaps we blunder on from one theory to the next, always trying to use our rational faculties so that gradually we approach the Ultimate Truth of granule architecture. Maybe so — but I doubt it. I find myself questioning the whole structure of our scientific reasoning, as well as the validity of any absolute concepts in science. Perhaps our most sacred scientific laws - gravitation, $e=m c^{2}$, the almost numinous Second Law of Thermodynamics — are merely temporary expedients or working hypotheses, eventually destined to be repealed or amended beyond recognition.

Let me go way back in history to trace the origins of our present scientific methodology, and to provide some background for my skepticism. Our whole Western culture (which includes our pattern of thinking as well as our moral, social 
and scientific values) has been profoundly influenced by the Greek philosophers of the 4th Century B.C., notably Plato, Aristotle and Euclid. In particular, two concepts have become so deeply embedded in our scheme of things that we call them "rational thinking," the basis of our scientific method. One of these is the idea of an infinite chain of cause-and-effect relationships; A produces B which in turn gives rise to $\mathrm{C} \longrightarrow$ and so on. A further assumption here is that a skilled mind can reason in either direction in this chain of events, to find the cause of what has happened, or to predict what will happen. This idea was expressed in a very sophisticated form in Euclid's geometry. The Greek philosophers, who were in a very real sense the originators of our scientific thought, considered this concept to be a dominant principle of existence. For example, Aristotle postulated an absolute cause-and-effect relationship for everything which had ever happened in the universe, tracing this chain of events inexorably back to the Prime Mover of original creation.

A second general concept which has permeated our thinking is a sort of categorical dualism, the existence and clash of opposite principles. For example, we scarcely ever think of gray as a color in itself, but rather as a mixture of black and white. Plato contended that an idea is either true or false, and today we do not even have words to express an in-between situation. In one of his more famous dialogues on the difference between knowledge and belief, Plato states that knowledge is true because it represents things as they actually are. I would call that kind of sophistry a prime example of argument-in-a-circle. These old Greeks were totally enthralled (and I believe totally anaesthetized) by the Absolute Truth of science and mathematics. They never even considered the possibility that both science and mathematics might not be knowledge of true things at all, but rather special languages for the communication of ideas, without any connotation of truth and falsity. Such ideas as $\sqrt{-1}$, or $1 / 0$, or $\int_{-\infty}^{+\infty}$ do these deserve labels of true or false, right or wrong? Or aren't they instead merely symbols in a language for the communications of thoughts and feelings, not very different in principle from the languages of poetry, music and art? It has been said that "beauty is in the beholder" __ more and more, it seems that the same can be said of truth. That which is sacred to one culture may be anathema to another.

These concepts were carried on through the Middle Ages by the "scholastic philosophers," who had the poor taste and worse sense to conceive of deterministic mathematics and scientific dualism as the binding forces on mankind. Thus human history was bound with the mathematical chains of predestination, and could offer no alternative in an after-life other than Heaven or Hell. Though dead infants were permitted to go to a lost Limbo.

Our modern thinking is the product of this background. Our vaunted Scientific Method would subject all things to experimental trial-and-error to establish truth or falsity, and would fix every event by scientific law into an inexorable pattern of cause and effect. I could elaborate at considerable length on this theme. 
Instead, I will take one beautifully illustrative case from modern political science: the determinism of history and the dualism of the dialectic, as so scientifically and boringly enunciated by Marx and Lenin:

"Dialectic is the study of how there can be and are identical opposites-

In mathematics: plus and minus, differential and integral

In mechanics: action and reaction

In physics: positive and negative electricity

In chemistry: the combining and dissociation of atoms

In social science: the class struggle......"

(from the notebooks of Nicolai Lenin)

So this is where the scientific method has led us?

To return to starch, let us suppose (and I think it is a safe proposition) that Kurt Meyer's model of granule structure is eventually proven to be erroneous. This probably would not happen all of a sudden by some major discovery, but rather by slow erosion of the theory, progressive alterations of concepts until no vestige of the original idea remained valid. This might perhaps be considered as scientific progress, a slow refinement or evolution toward some absolutely true concept. However, there are certain arguments against this viewpoint:

1. The supplanting theory would then undergo the same gradual erosion toward eventual falsity, and so would every succeeding theory.

2. The Meyer theory during its heyday has been a highly useful tool in investigative research. From an absolutist standpoint, it is difficult to understand how a concept which is eventually proven erroneous can ever give rise to anything valid or useful.

3. After all, each theory is the personal creation of flesh-and-blood scientists, each of whom is egotistically involved with his cherished interpretations. Hence the viewpoints in science are inextricably entangled with human personalities and emotions, and there is really no such thing as a dispassionate observer.

So I must reject the idea of progress toward ultimate truth or the unadulterated quest for knowledge as the primary motivations of scientific research. Having thus denied any such idealized function of science, what then can be its purpose? I suggest the objective of practical usefulness as sole and sufficient justification. This may take a variety of forms: the profit return from invention and marketing; the sense of power and prestige conveyed by control over natural phenomena; the economic, social or political advantage over one's contemporaries or adversaries. For example, I believe that the space race between the U.S. and Russia is motivated on both sides entirely by these considerations, and not by any altruistic idea of human benefit or impersonal thought of increasing our total knowledge. In no sense do I either damn or extol these motivations of power and profit — I am merely trying to view the total picture with some degree of perspective. And in our western dollar-oriented industry, the urge for power is probably more important than the profit motive. This really underlies the growth-by-acquisition trend, a pathway to the coveted goal of a billion dollars per year of gross sales. I recall one company 
which entered this "billion-dollar club" proudly flaunted its achievement in each annual report, carefully minimizing the unpleasant fact that its net profits were steadily declining. With companies as with nations, the lust for power is dominant, frequently to the detriment of both practical efficiency and any humane values. Thus top management will hire a professional "hatchet man" to slash away its time-worn employees, with no consideration for their profound experience'and long years of service. This would not be tolerated in the more humane Japan.

Thus I am assuming that the only criterion of value in any scientific activity is the practical utility of results. I must make a second assumption which is difficult to defend: that every scientific observation (no matter how remote or obscure) will eventually have some potentiality for practical utility. When in 1898 the Curies isolated radium from pitchblende, their studies on this extremely rare element might with good reason have been regarded as utterly blue-sky and impractical indeed, a foolish waste of time, effort and money.

So I think we can classify all research according to the time required for realization of utility and profit. Suppose we take some examples (both real and imaginary) from the starch industry:

1. The fire-department type of research to meet a critical emergency in production operations: for example, the sudden and inexplicable development of filtration problems with starch conversion liquors. What can be done fast by process modification to get out of trouble and back into production? Simultaneously, a crash project might be undertaken to isolate and identify the trace material which is plugging the filters or creating haze problems. The answers must be obtained in a minimum of time, and any such work obviously takes priority. Every company encounters occasional trouble spots of this sort; however, a chronic state of emergencies, either in a business organization or in national affairs, is a danger signal that something is inherently wrong.

2. Short-range development of new products or processes with definitely-defined objectives. This is the most familiar type of industrial investigation, and is best exemplified by the continuous effort to improve existing products for a well-established market. Take for instance the development of a better starch for use as a thickening agent in salad dressings. We think we know most of the requisite characteristics 'of such a starch: food acceptability, a high and stable viscosity, resistance toward shear, a short-textured consistency and the absence of any elasticity. in the final product, resistance to acidity of the system, a certain opacity to enhance color appeal, etc. Can all these objectives be realized with a single starch, or do we need a blend? With a well-defined objective and a clearly delineated route of investigation, the time required for such a project should be measured in months, not years. Longer development time is usually undesirable for several reasons: (a) competition may run away with the market while your product is still in a prolonged laboratory-development stage, (b) statistically it makes better sense to divide the total development effort among a variety of shortrange items, and (c) the sales-life of the final product may be only several years before competition catches up. 
3. Long-range development research with general objectives. The primary purpose here is to provide new consumer markets 5 or 10 years hence. In general, two types of developmental research are encountered:

(a) We have a novel or unusual material or phenomenon-what profitable enduses can be found for it?

(b) We have conceived a potentially-profitable end-use-what material or process can be developed to fulfill this specific need?

As an example in the first category, commercial quantities of the linear fractions from corn and potato starches are now available, as well as high-amylose corn starch containing as much as $80 \%$ of linear material. What useful and profitable applications can be found for this novel linear polymer? As a possible instance, we find that small amounts of the linear fraction will cause concentrated solutions of sodium hydroxide to set up to rigid heat-reversible gels. Curiously enough, this does not happen with either lithium or potassium hydroxides. Can markets be found to utilize this peculiar behavior? An excellent example in the second category is in the starch-sizing of paper. Most magazines are printed on glossy paper surfacecoated with clay or other opacifier in an adhesive matrix of pasted starch. Such a surface will permit sharp high-speed printing of photographic reproductions and color illustrations. However, there is one very objectionable feature-the inorganic opacifier markedly increases the weight of the paper and thus raises the mailing cost of the final magazine. Hence considerable research is being conducted on starches and other colloidal agents to produce a foam coating, in which the opacifying agent is simply air emulsified to a bubble size of perhaps a few tenths of a micron. Such an opacifier would have no weight whatsoever on the post office scales. Because the investigational routes are so completely uncharted, development research of this kind requires the utmost in imaginative thinking and ingenuity-plus plenty of time.

4. Fundamental research. This might be defined as long-term investigation without definitely stipulated goals, except the general principle of discovering new phenomena and developing new concepts. I am sure that this type of research pays off in the long run, though the profits may not be enjoyed by the present generation of stock-holders. An excellent and actual example is the study of the composition of starch, initiated by myself in 1937. Over a period of almost 15 years, this work developed the first separation of authentic linear and branched polymers of starch, made substantial progress in elucidating the chemical structures and physical characteristics of these components, and identified the physicochemical differences between various natural and modified starches. While these investigations cannot be appraised in terms of direct profits, nevertheless they triggered a wide variety of developments on waxy and high-amylose starches, as well as the ether and ester derivatives of starch. Unfortunately, the cereal, milling and baking industries have been quite laggard in long-range fundamental research, though there are numerous areas in which new and potentially useful concepts are needed, for example, on the anatomy of cereal grains, the chemical and physical changes during germination and growth of the seed, the submicro-structure of bread, and especially the physico- 
chemical reactions of cereal proteins. I strongly believe that $5-15 \%$ of the total scientific budgets of the cereal industry might advantageously be allocated to this type of research, with no concern and no pressure for immediate profits.

The primary essential for successful research is people with curious and ingenious minds, working with their hands at the laboratory bench, and sufficiently aware of themselves and their work to recognize the new and different. Let me cite an example from the starch industry. In textile and paper manufacture, the batch cooking of starch sizing is being replaced by continuous cooking techniques, whereby an aqueous starch slurry and high-pressure steam are judiciously mixed to effect instantaneous pasting of the starch at temperatures around or above $300^{\circ} \mathrm{F}$. In one particular textile mill, a corn starch paste cooked in this fashion was allowed to stand and cool. It was then found to be unusable as a sizing due to the presence of insoluble "granules" showing an interference cross under polarized light. The mill operators immediately concluded that the corn starch could not have been properly gelatinized by the steam-jet cooker. But the more intimate experience of the starch chemists challenged this conclusion on several grounds. How could a granule survive the super-temperature of jet-cooking without undergoing swelling? Moreover, these granules had not been apparent in the freshly-cooked paste. And so this brought to mind a long-forgotten publication by Beijerinck in 1915, describing similar spherocrystals which formed during the aging of autoclaved starch pastes. Subsequent isolation of these "granules" showed them to be a crystallized form of the linear fraction of the corn starch. Also so this chain of circumstances finally led to the development of a commercial method for fractionating starch. Here we have all the elements essential to scientific discovery: minds keenly alert to recognize the unusual, the persistent curiosity to delve into the "how" and "why", an intimate acquaintance with the past literature, and finally the technical competence to establish the practicality of the discovery.

But we insist on cluttering up this relatively simple research pattern with all sorts of impedimenta and operational gadgetry, primarily because we keep forgetting the personal nature of scientific discovery. For example, the proper function of research management is decision-making: the selection of the most promising areas for investigation, the choice of personnel and the prudent allocation of funds, the practical mechanics of translating laboratory discoveries into useful benefits. Yet too frequently, research management tries to "master-mind" discovery, to do the imaginative and creative thinking, and to adopt a defensive attitude in "justifying" the research budget. There seems to be a naive belief in many industry circles that organization, "know-how," and the expenditure of money are the only requisites for successful research. Thus the organization chart is regarded as the road-map of discovery. The bigger the institution, the more complex and ramified is this chart, until eventually the whole hierarchy may look very much like the U.S. Army. Too often the first-rate research minds are "promoted" to administrative duties, and the actual bench-work is left to second-stringers. Here again, we forget that inspirations do not normally occur to administrators buried in paper-work, but rather to the 
creative co-functioning of mind and hands on the part of the bench chemist, who sees and recognizes something new and different.

Then we build a multi-million dollar complex of research laboratories, and fill these with the latest and most expensive electronic devices. I am not decrying good equipment and good working facilities-I am simply objecting to the substitution of unimaginative machines for human rninds. I have seen far too much sloppy data in published form, where correlation between factors could only be derived with a computer and statistical evaluation.

We seem to be over-impressed with the status symbol of the graduate academic degree, particularly the $\mathrm{Ph}$. D. A doctorate simply implies that the individual has been exposed to original research; it does not guarantee that the experience has left any lasting mark. I have seen bachelors of science who out-shone many Ph. D.s in the originality of their thinking and the ingenuity of their experimentation-and I have seen Ph. D.s who might perhaps do a creditable job of teaching high-school science. I realize that curiosity and ingenuity are innate qualities of the mind, and cannot be learned in the classroom. Nevertheless, I have wondered just what the university does teach at the graduate level, and whether this function might not be performed just as well or even better by a good trade school.

Also, I deplore the quality of research papers which are published in the scientific journals. Perhaps half of these might be consigned to the nearest wastebasket with no great loss to the world. In far too many cases, these publications have no valid objectives, the planning of experimentation shows no imagination, the actual work is sloppy and careless, and the results are hackneyed and meaningless. It seems a shame that good research funds should be wasted on this sort of rubbish. Yet publication is much sought after, particularly in universities and government laboratories, and advancement too frequently depends on the quantity rather than the quality of publications.

Let me summarize briefly. I have argued that we should break away from the antiquated dualism of true-or-false in science, and recognize that practical values are the only criterion for appraising research. Science is not (and probably will never be) an orderly correlated system of truths and laws, but rather an ever-changing language for the communication and proliferation of ideas and observations. Scientific theories have merit only if they lead on to new ideas and new discoveries-and it makes little difference if the guiding theory is "right" or "wrong." No phenomenon has a complete and ultimate explanation. Research can be classified in terms of the time required for results to become practical and profitable, ranging from the rushorder solving of emergency situations to the long-range fundamental research for new phenomena and new ideas. The only absolute requirement for successful research is a curious and ingenious mind, and the self-imposed clutter of status symbols, implementation aids and organizational gadgetry has little real value in producing discoveries.

In conclusion, I can find only one definition of the "scientific method" which appeals to me. This was expressed by Ernest Nagel, of the Philosophy Department 
of Columbia University. He says:

"What is loosely called (the) scientific method is generally a habit of workmanship that skilled investigators possess, and not a codified set of principles which they explicitly acknowledge".

It's that simple, and everything else is merely window-dressing. 